PSYCBOMETRIKA - VOL. 19 , No. 3

BEPTEMBER, 1954

\title{
CHANGES IN FACTOR STRUCTURE OF A COMPLEX PSYCHOMOTOR TEST AS A FUNCTION OF PRACTICE
}

\author{
Edwin A. Fleishman and Walter E. Hempel, Jr. \\ AF PERSONNEL AND TRAINING RESEARCH CENTER* \\ LACKLAND AIR FORCE BASE \\ SAN ANTONIO, TEXAS
}

Scores obtained at eight different stages of practice on the Complex Coordination Test together with scores on 18 reference tests were subjected to a Thurstone Centroid Factor Analysis. Nine meaningful factors were identified in the experimental battery. The results indicated considerable, but systematic, changes in the factor structure of the Complex Coordination Test as practice on the task was continued. The test became less complex (factorially) as practice was continued. Moreover, there was a change in the nature of the factors contributing variance at early and later stages of practice. Implications of the findings are related to certain problems of learning theory, psychomotor test development, and criterion analysis.

\section{Introduction}

In some previous studies $(3,8)$ evidence was found that even during the short time of administration of a single psychomotor test, the ability or abilities sampled may shift materially in importance. This was indicated by variations in the factor patterns and in the weights of particular factors at various stages of practice on the particular tests investigated. Similar results also had been found earlier in factorial studies of extended practice on printed tests $(2,5,13,15,16,17)$. The problem appears more crucial in the psychomotor area, however, since measures of such skills typically show quite rapid and extensive improvement with even brief amounts of practice. It then becomes important to establish what abilities are being sampled at different stages in performance as practice is continued on particular psychomotor tasks. Such knowledge would have implications for future test development in this aptitude area as well as for questions concerning the processes involved in the learning of complex perceptual-motor skills.

*Skill Components Research Laboratory. The opinions or conclusions contained in this report are those of the authors and do not necessarily reflect the views or indorsement of the Department of the Air Force.

The writers are indebted to Dr. Jack A. Adams for the basic data on which this analysis is based. 


\section{Purpose}

The study to be reported here was designed to investigate further the nature of these changes in ability patterns which seem to occur as practice continues on a complex psychomotor test. More specifically, an attempt was made to identify the factors involved at different stages of performance on a criterion psychomotor task, through the inclusion of well-established reference variables in the analysis. The interpretation of the nature of the factors involved at various practice stages has been limited in the earlier studies by the absence of such sufficiently well-defined reference variables. Moreover, in the present study practice on the criterion task was continued over a considerably longer time period than had been investigated previously.

The analysis also was aimed at identifying (1) the stages of practice in which the task is most complex (in terms of the number of abilities sampled), (2) the stages at which systematic changes in factor structure occur, (3) the stage at which the factor structure becomes stabilized, (4) the relative importance of "motor" versus "non-motor" factors at early and late stages of practice.

\section{Method}

A factor analysis of performance at various stages of practice on the criterion task together with selected reference tests was carried out by the Thurstone Centroid Method (12). The analysis is based on a sample of 197 basic airmen tested at Lackland Air Force Base. Variables in the analysis are described below.

The Criterion Practice Task: The Complex Coordination Test, Model E, was the criterion task learned. The test has been described in detail elsewhere by Melton (8). Essentially, the subject is required to make complex motor adjustments of an airplane-type stick and rudder in response to successively presented patterns of visual signals. A correct response (movement of stick and rudder controls to proper positions) is not accomplished until both the hands and feet have completed the appropriate movements. A new pattern appears as each correct response is completed. Score is the number of correct responses completed in a given test period. This task was selected since it has a high learning ceiling, shows no appreciable decrease in relative variability between subjects as practice is continued, and was suspected to be factorially complex. The acquisition curve for this task has been presented elsewhere $(1,10)$.

Practice on the test was continued over 64 two-minute trials. For each subject, testing was accomplished over a two-day period, each day involving a morning and afternoon session. Each session included 16 trials separated by one-minute rest intervals. 
Scores obtained during the following eight segments of the total practice period were selected for inclusion in the present analysis:

1. Stage 1-Trials 1-5.

2. Stage 2-Trials 12-16.

3. Stage 3-Trials 17-21.

4. Stage 4-Trials 28-32.

5. Stage 5-Trials 33-37.

6. Stage 6-Trials 44-48.

7. Stage 7-Trials 49-53.

8. Stage 8-Trials $60-64$.

These stages of practice on the criterion task, which represent the first eight variables in the present analysis, include the first and last ten minutes of practice during each of the four testing sessions.

Test Variables: The following printed tests were included in the analysis. They have been described in detail by Guilford (7).

9. Numerical Operations-II. A highly speeded test requiring simple subtraction and division operations.

10. Dial and Table Reading. A series of instrument dials and mathematical tables from which information must be read accurately and quickly.

11. Mechanical Principles. Pictorial items which require the comprehension of principles and mechanisms, such as leverage, and rotation and transformation of motion, involved in the action and uses of various mechanical devices.

12. General Mechanics. Verbally presented items of practical mechanical information dealing with the use and operation of familiar mechanical methods and devices.

13. Speed of Identification. Pictorial items in which the silhouette of an object must be identified quickly when it is rotated and imbedded in a group of similar silhouettes.

14. Patlern Comprehension. A series of drawings requiring visualization of relationships between components of solids and their unfolded fat projections.

15. Visual Pursuit. From a series of mazes of irregularly curved lines, the task is to trace each line visually from its beginning to its proper termination point.

16. Decoding Test. A series of short words, written in a code of flag symbols, must be decoded by relating the position of repeated symbols to the positions of repeated letters.

17. Instrument Comprehension. For each item which presents views of cockpit instruments the examinee must determine the proper position or orientation of an airplane.

18. Spatial Orientation. From a large aerial photograph or map, the examinee must find the area that matches each of a series of amall photographs.

19. Speed of Marking. The examinee marks an IBM answer sheet in the indicated spaces as rapidly as possible.

20. Log Book Accuracy. On an IBM answer sheet, the examinee marks as quickly and accurately as possible the proper letter indicated in a separate test booklet. 
The following apparatus tests were included. They have been described in detail by Melton (8).

21. Rotary Pursuit. The examinee attempts to keep a stylus in contact with a small rnetallic target set in a rapidly revolving disk.

22. Plane Control. The task is to make compensatory adjustments of stick and pedal controls in order to keep a model plane straight and level.

23. Discrimination Reaction Time. The examinee manipulates one of four toggle switches as quickly as possible in response to a series of visual stimulus patterns differing from one another with respect to the spatial arrangement of their component parts.

Three additional apparatus tests which have not been described elsewhere were also included.

24. Nut and Boll. The task is to insert and fasten as many nut and bolt assemblies as possible through a series of holes in an upright metal plate.

25. Reaction Time. A series of reactions in which the examinee must move his hand six inches as quickly as possible to a switch in response to a light stimulus which appears at varying intervals.

26. Rate of Movement. The examinee's task is to break the beams between a series of photoelectric cells, one after another, by making scalloped movements of his hand as rapidly as possible.

\section{Results}

Interpretation of Factors: The intercorrelations (Pearson product-moment) among the 26 variables are presented in Table 1 . Ten factors were extracted from this matrix. The complete centroid factor matrix obtained is presented in Table 2. Orthogonal rotations were made using Zimmerman's graphical method (20). Table 3 presents the orthogonal solution of rotated factor loadings obtained using the criteria of simple structure and positive manifold.

Variables having orthogonal projections of .30 or larger on the rotated axes were considered in defining a factor. However, loadings of .25 or higher are considered significant (see Table 3 ). Interpretations given certain of the factors have drawn on several previous Air Force analyses $(6,7,9,18,19)$.

Factor $I$ is common only to stages of practice on the criterion task, the Complex Coordination Test. Moreover, it can be seen (Table 3) that the loadings increase progressively through stage 4 and then remain at about the same level through the remaining practice stages. For the present, this factor is called the Complex Coordination Test Specific.

Factor $I I$ is common to all eight stages of practice on the Complex Coordination Test and to the Rotary Pursuit Test. It also shows a low loading in the Plane Control Test. This factor appears to be the same as the factor called Psychomotor Coordination in many previous analyses of the Aircrew Classification Batteries. It has consistently been identified in all analyses which have included the Rotary Pursuit and Complex Coordination Tests (the latter represented by stage 1 in the present analysis). The factor has 
EDWIN A. FLEISHMAN AND WALTER E. HEMPEL, $\boldsymbol{J} R$.

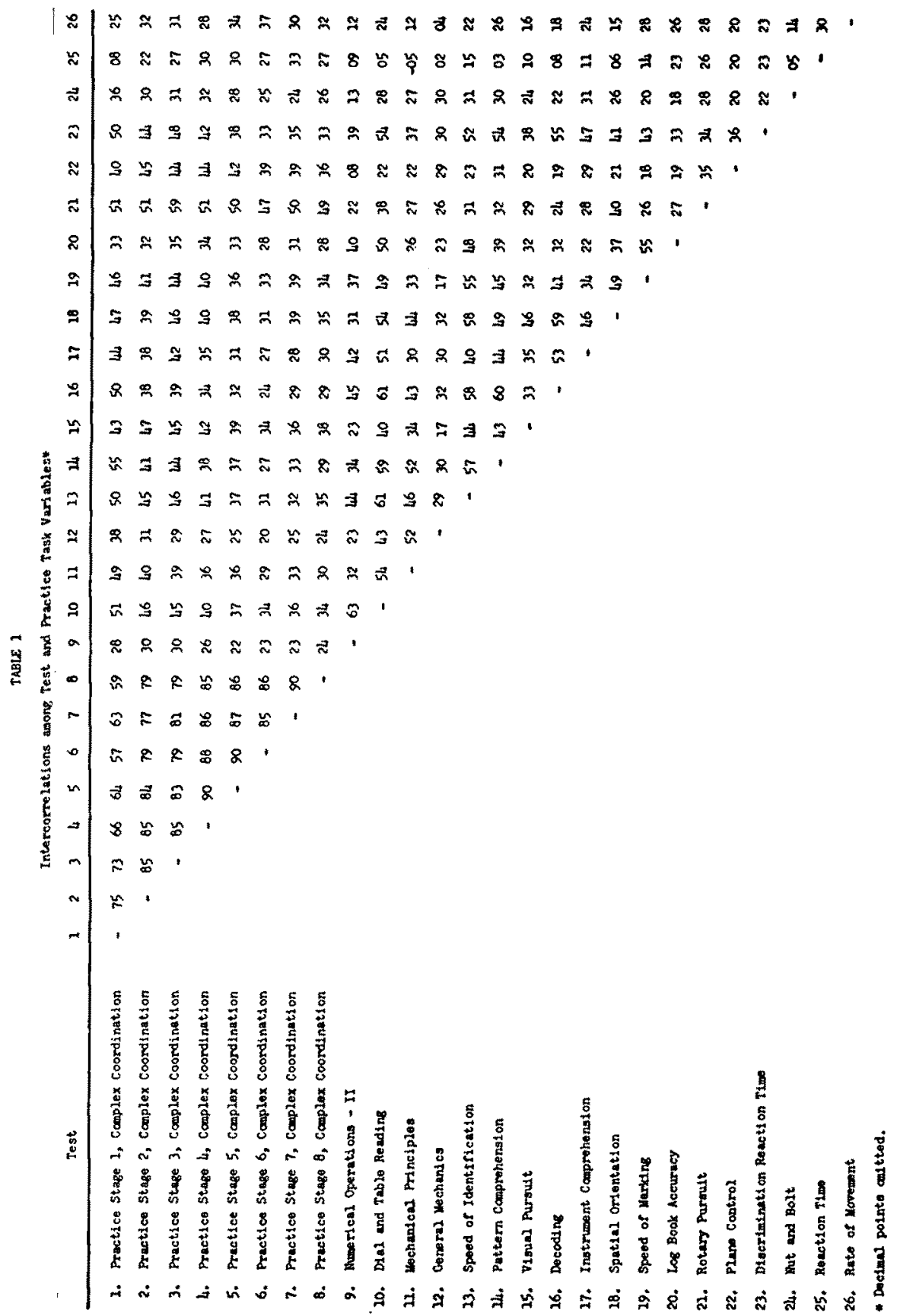


been defined rather broadly as representing either coordination of the large muscles of the body, in movements of moderate scope, or coordination of such movements with the perception of a visual stimulus.

The present analysis indicates that this factor is most adequately sampled in stages 2 and 3 of the Complex Coordination Test, but that it maintains its importance at all stages of practice on the test.

Factor $I I I$ is defined primarily by the two apparatus tests, Reaction Time and Rate of Movement, which are practically pure measures of this factor. Significant loadings are also evidenced by the Rotary Pursuit, Plane Control, and Discrimination Reaction Time Tests. It seems clear that this factor may be defined as Rate of Movement. It is possible that this factor extends beyond rate of arm movement to leg and feet movements as well.

With respect to the importance of this factor at different stages of practice on the criterion task, we may again refer to Table 3 . It can be seen that the first stage of practice shows an insignificant loading on this factor, but that subsequent stages show a progressive increase to a point later in training, where the loadings become stabilized.

Factor IV appears to be a Spatial Relations factor. Most direct evidence for this interpretation is found in the significant loadings of the Discrimination Reaction Time, Instrument Comprehension, Complex Coordination, and Dial and Table Reading Tests, which have consistently been found saturated with this factor. The loadings of the remaining tests on this factor are not inconsistent with the interpretation of this factor as primarily Spatial in nature. A possible exception is the relatively high loading of the Decoding Test. It is, thus, possible that Factor IV may be a composite of Reasoning and Spatial Relations factors. However, the remaining tests are primarily spatial in nature. This factor seems to involve the ability to relate different responses to different stimuli, where either stimuli or responses are arranged in spatial order. Emphasis in this Spatial factor appears to be on decision as to direction of movement.

It can be seen in Table 3 that this factor is most importantly involved in the first stage of practice on the Complex Coordination Test and is not found in later stages of practice.

Factor $V$ is defined primarily by the Visual Pursuit, Speed of Identification, and Spatial Orientation Tests, and also by Instrument Comprehension and Dial and Table Reading. This factor is readily defined as Perceptual Speed. It involves the rapid comparison of visual forms and the notation of similarities and differences in form and detail.

It is found in the Complex Coordination task only to a low degree with a loading of .25 or above only in certain of the earlier stages of practice.

Factor VI is defined principally by Pattern Comprehension and Mechanical Principles and also by Decoding, Spatial Orientation, and Dial and Table Reading. The tests with the two highest loadings have consistently 
TAELS 2

Centrofd Factor Loadings of Test and Practice Task Variableg*

\begin{tabular}{|c|c|c|c|c|c|c|c|c|c|c|c|c|}
\hline & Teqt & $I$ & II & III & IV & $y$ & VI & VII & VIII & IX & $x$ & $h^{2}$ \\
\hline $\begin{array}{l}1 . \\
2 . \\
3 . \\
5 . \\
6 . \\
7 . \\
8 . \\
9 . \\
10 . \\
11 . \\
12 . \\
13 . \\
14 . \\
15 . \\
16 . \\
17 . \\
18 . \\
29 . \\
20 . \\
21 . \\
22 . \\
23 . \\
24 . \\
25 . \\
26 .\end{array}$ & 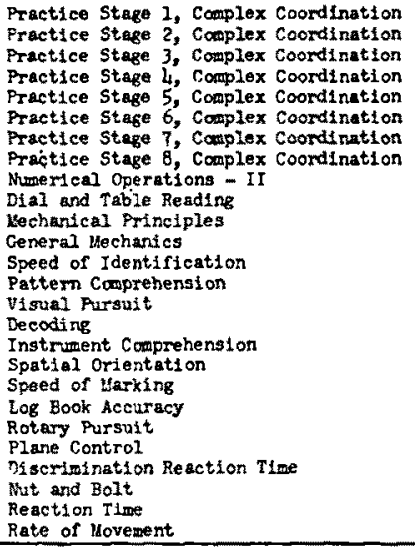 & $\begin{array}{l}79 \\
83 \\
85 \\
83 \\
80 \\
75 \\
77 \\
76 \\
49 \\
71 \\
56 \\
45 \\
68 \\
65 \\
55 \\
62 \\
57 \\
64 \\
60 \\
53 \\
60 \\
47 \\
64 \\
41 \\
27 \\
39 \\
\end{array}$ & $\begin{array}{r}04 \\
34 \\
33 \\
43 \\
48 \\
52 \\
48 \\
48 \\
-35 \\
-444 \\
-29 \\
-20 \\
-37 \\
-35 \\
-11 \\
-41 \\
-25 \\
-31 \\
-23 \\
-21 \\
17 \\
16 \\
-22 \\
-07 \\
24 \\
13 \\
\end{array}$ & $\begin{array}{r}20 \\
12 \\
07 \\
13 \\
13 \\
07 \\
09 \\
14 \\
-21 \\
-08 \\
33 \\
13 \\
02 \\
07 \\
12 \\
06 \\
-08 \\
14 \\
-14 \\
-25 \\
-10 \\
-07 \\
-15 \\
10 \\
-39 \\
-31 \\
\end{array}$ & $\begin{array}{r}-17 \\
03 \\
01 \\
13 \\
10 \\
14 \\
15 \\
15 \\
21 \\
07 \\
-14 \\
-26 \\
15 \\
-17 \\
10 \\
-03 \\
-17 \\
07 \\
24 \\
30 \\
-13 \\
-29 \\
-12 \\
-18 \\
03 \\
-06 \\
\end{array}$ & $\begin{array}{r}-09 \\
-07 \\
-08 \\
02 \\
03 \\
09 \\
06 \\
07 \\
22 \\
22 \\
23 \\
36 \\
-17 \\
-14 \\
-18 \\
-17 \\
-09 \\
-11 \\
-13 \\
05 \\
04 \\
-03 \\
-18 \\
08 \\
-05 \\
-08 \\
\end{array}$ & $\begin{array}{r}-11 \\
-05 \\
-07 \\
04 \\
10 \\
13 \\
08 \\
11 \\
19 \\
10 \\
-01 \\
-15 \\
-08 \\
05 \\
-08 \\
27 \\
19 \\
-08 \\
-14 \\
-22 \\
-17 \\
-07 \\
12 \\
-10 \\
03 \\
06 \\
\end{array}$ & $\begin{array}{l}-13 \\
-16 \\
-05 \\
-06 \\
-05 \\
-02 \\
05 \\
10 \\
-11 \\
-10 \\
-12 \\
-06 \\
-03 \\
-10 \\
14 \\
-05 \\
14 \\
20 \\
-08 \\
-08 \\
15 \\
-09 \\
-16 \\
15 \\
10 \\
08 \\
\end{array}$ & $\begin{array}{r}13 \\
08 \\
13 \\
-04 \\
-10 \\
-09 \\
05 \\
04 \\
19 \\
12 \\
-10 \\
07 \\
-10 \\
-17 \\
-04 \\
17 \\
15 \\
08 \\
-07 \\
-18 \\
13 \\
-03 \\
03 \\
-09 \\
-11 \\
-21 \\
\end{array}$ & $\begin{array}{c}07 \\
14 \\
04 \\
-09 \\
-01 \\
05 \\
-06 \\
-02 \\
09 \\
14 \\
07 \\
-22 \\
-05 \\
11 \\
11 \\
-12 \\
-04 \\
-10 \\
-05 \\
-04 \\
10 \\
-13 \\
-07 \\
-03 \\
-19 \\
14 \\
\end{array}$ & $\begin{array}{r}10 \\
-13 \\
-05 \\
-12 \\
01 \\
-02 \\
11 \\
02 \\
-30 \\
04 \\
05 \\
-05 \\
-03 \\
15 \\
-13 \\
14 \\
-15 \\
15 \\
19 \\
11 \\
08 \\
-09 \\
-10 \\
-11 \\
-04 \\
13 \\
\end{array}$ & $\begin{array}{l}76 \\
90 \\
87 \\
93 \\
92 \\
69 \\
90 \\
87 \\
59 \\
80 \\
62 \\
53 \\
66 \\
67 \\
43 \\
72 \\
53 \\
63 \\
57 \\
58 \\
50 \\
38 \\
58 \\
27 \\
34 \\
36 \\
\end{array}$ \\
\hline \multicolumn{2}{|c|}{$2 a^{2} / k$} & 42 & 10 & 03 & 02 & 02 & $O 2$ & 01 & 01 & ol & 01 & \\
\hline
\end{tabular}

* Decional points ond tied.

TABLE 3

Rotated Factor Loadings of Test and Practice Task Variablesr-

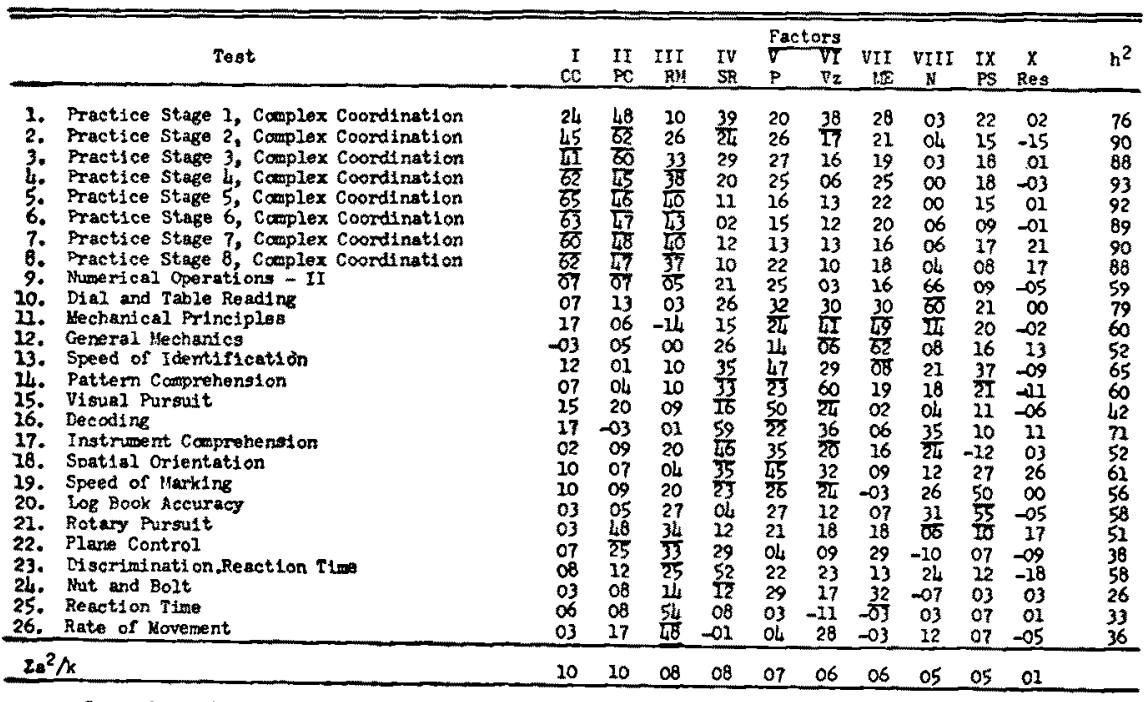

* Decinar points conitted. 


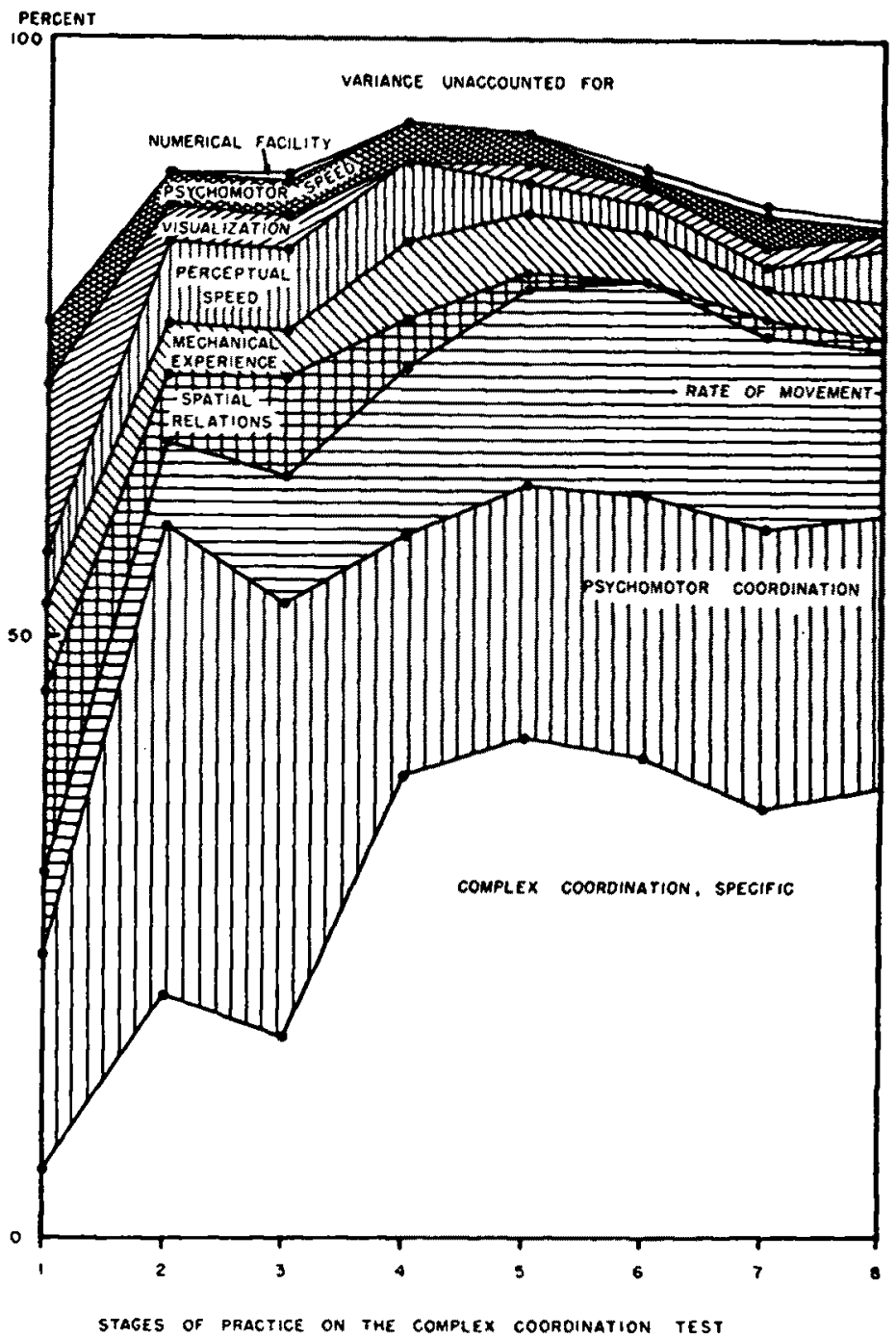

Fig. 1.-Percentage of variance (shaded area) represented by each factor at different stages of practice on the Complex Coordination Test.

after certain amounts of practice are likely to depend more on certain abilities and less on others than they did initially.

Learning theorists have given relatively little attention to the relationships of individual differences to the principles of learning. It is therefore difficult to relate the results of the present study to any specific learning theory. However, a few investigators have given some attention to the problem. Reynolds (10), for example, found decreasing correlations between 
printed tests and a psychomotor test as practice was continued on the latter test. He hypothesized that the subject's early performance is to a great extent a function of previous experience and therefore reflects a number of abilities. As learning continues, the subject's performance depends more upon his experience with the particular task employed. To an increasing extent, he learns the specific responses required by the task. The abilities or aptitudes associated with these specific habits being acquired, it is hypothesized, are progressively restricted in extent and generality. This view appears related to Seashore's "work methods" hypothesis (11) as well as to some of the findings of Woodrow (cf. 14). In factor analyses of extended practice on printed tests, Woodrow found that loadings of practice gains scores are often specific to the particular test.

Some of the data of the present study are not inconsistent with these previous interpretations. For example, it has been noted that as practice was continued on the Complex Coordination Test there was a progressive increase in loadings on a factor common only to the test itself. Also consistent with these hypotheses is the shrinkage in the number of factors contributing to performance as practice is continued. However, such "specificity hypotheses" imply that a sizable portion of the between-subjects variance observed at advanced levels of proficiency in a task is not ascribable to variation along any other dimension other than that established by the task itself.

At present, however, this view is regarded as overly pessimistic, especially with respect to the problem of predicting more advanced and terminal proficiency in psychomotor skills. It is felt, rather, that the problem is one of identifying what variables predict such advanced proficiency levels. The present study indicates that it is possible to isolate variables which predict performance at advanced stages. For example, the Rate of Movement and Psychomotor Coordination factors were found to contribute a sizable portion of the variance at advanced stages. The factor found specific to only the trials of the practice task may be broken down still further, with additional experimentation including other variables. Perhaps the most encouraging results regarding the predictability of advanced stages of psychomotor performance are found in recent work by Adams (1). He found that a combination of certain test measures yielded a multiple correlation with advanced levels of performance on this criterion task that exceeded the correlation between the first and last stages of practice on the task itself. This gives further indication that the abilities sampled at late stages of practice on a psychomotor task are not necessarily specific to the task, but may represent common factors definable by other test variables.

For the present, performance at any stage of practice is regarded as determined by a set of cooperating but independently variable abilities. This view is similar to that proposed by Woodrow (13). Continuation of practice on the task does not result in an equal increase or decrease in 
favorableness for the operation of all the cooperating determining factors contributing to individual differences. For example, in the early stages of performing the task, learning the spatial relationships of the different stimuli to the different responses may be an important contributor to individual differences. Once this is learned to a sufficiently high degree and no further improvement in this skill is possible (or necessary), within the limits of the task, other features of the task may assume increasing importance (e.g., completing the proper sequence of movements quickly). However, the important point is that earlier in the learning period the use of certain skills is a minimal but necessary requirement for achieving a certain amount of progress in performing the task. At this stage, individual differences in these skills affect the rise in the acquisition curve. Later in training, individual differences in other combinations of skills may be the chief contributors to performance. The rise in the performance curve may be considered to be a resultant of systematic transformations in the particular combination of abilities contributing variance at different stages of practice.

\section{Implications}

The results of the present study would appear to have certain implications for test development in this aptitude area and for certain problems of criterion analysis. For example, the fact of changes in factor pattern with practice points up the importance of establishing for such tests what abilities are contributing variance at different stages of performance on the test. The problem appears especially crucial in regard to psychomotor tests which are included as parts of larger, rather comprehensive classification batteries. The need for excluding from such apparatus tests variance measurable by printed tests has been pointed out earlier (4). Knowledge about factorial content at different stages should enable one to specify how much practice to give on such tests in the operational test situation in order to reach the stage of performance at which it would be most desirable to score the test; that is, performance up to a given stage would be purely "practice" for the subject and scoring would begin after this stage is reached and for a specific period. This would include the stage at which (1) the factors measured by the test which are also measured by the other tests are at a minimum, and (2) the loadings of the remaining valid factors in the test are at a maximum. This would presumably maximize the unique contribution of such tests to the predictive power of the total battery.

The findings of the present study are indirectly related to certain problems of criterion development in operational situations. For example, testing programs are most often evaluated against more immediate criteria of proficiency in lieu of more intermediate or ultimate criteria of performance. Thus, the tests used in the Aircrew Classification Battery were, without exception, designed to predict the success of candidates in training for the 
specialty to which they were assigned. Similarly, in the industrial situation, test validation is often conducted against proficiency at relatively early stages of training in place of validation against the more elusive on-the-job criteria. Aside from the several reasons supporting the use of the more immediate criteria (and there are many), the data of the present study at least suggest the ieed for criterion analyses of more advanced levels of proficiency, especially in jobs emphasizing perceptual-motor skills. It is quite conceivable that the abilities contributing to individual differences in earlier stages of skill attainment in such jobs may be somewhat different than those contributing variance at more advanced and terminal levels of proficiency. Thus, the relative size of test regression weights determined against immediate criteria may be expected to shift, when the same battery is evaluated against more advanced criteria. Similarly, tests rejected from the battery because of insignificant validity against immediate criteria may have turned out to possess considerable validity for advanced levels of proficiency. As a corollary of these possibilities, there is a need for developing tests which predict individual differences at more advanced and terminal levels of proficiency in such situations.

\section{REFERENCES}

1. Adams, J. A. The prediction of performance at advanced stages of training on a complex psychomotor task. San Antonio, Tex.: Human Resources Research Center, Lackland Air Force Base, December 1953. (Research Bulletin 53-49.)

2. Anastasi, A. The influence of specific experience upon mental organization. Genetic Psychol. Monogr., 1936, 18, 245-255.

3. Fleishman, E. A. A factor anslysis of intra-task performance on two psychomotor tests. Psychometrika, 1953, 18, 45-55.

4. Fleishman, E. A. Testing for psychomotor abilities by means of apparatus tests. Psychol. Bull., 1953, 60, 241-262.

5. Greene, E. B. An analysis of random and systematic changes with practice. Psychometrika, 1943, 8, 37-52.

6. Guilford, J. P., Fruchter, B., and Zimmerman, W. S. Factor analysis of the Army Air Forces Shepard Field Battery of experimental aptitude tests. Psychometrika, $1952,17,45-68$.

7. Guilford, J. P. (Ed.) Printed Classification Tests. AAF Aviation Psychology Program Research Report No. 5, 1947.

8. Melton, A. W. (Ed.) Apparatus Tests. AAF Aviation Psychology Program Research Report No. 4, 1947.

9. Michael, W. B. Factor analysis of tests and criteria: a comparative study of two AAF pilot populations. Psychol. Monogr., 1949, 63, No. 298.

10. Reynolds, $B$. The effect of learning on the predictability of psychomotor performance. J. exp. Psychol., 1952, 44, 189-198.

11. Seahore, R. H. Work and motor performance. In Stevens, S. S. (Ed.) Handbook of experimental psychology. New York: Wiley, 1951.

12. Thurstone, L. L. Multiple-factor analysis. Chicago: Univ. Chicago Press, 1947.

13. Woodrow, H. Factore in improvement with practice. J. Psychol., 1939, 7, 55-70.

14. Woodrow, H. The ability to learn. Psychol. Rev., 1946, 63, 147-158. 
15. Woodrow, H. The application of factor-analysis to problems of practice. J. gen. Psychol, $1939,21,457-460$.

16. Woodrow, $\mathrm{H}$. The relation between abilities and improvement with practice. $J$. educ. Psychol., 1938, 29, 215-230.

17. Woodrow, $H$. The relation of verbal ability to improvement with practice in verbal tests. J. educ. Psychol., 1939, 30, 370-382.

18. Zachert, Virginia, and Friedman, G. Factorial comparisons of two Airerew Classification Batteries with and without the variable of previous flying experience. San Antonio, Tex: Human Resources Research Center, Lackland Air Force Base, April 1952. (Research Bulletin 52-16.)

19. Zachert, Virginia, and Ivens, F. C. Comparative factor analyses of tests using three different scoring techniques. San Antonio, Tex.: Human Resources Research Center, Lackland Air Force Base, August 1951. (Research Bulletin 51-16.)

20. Zimmerman, W. S. A simple graphical method for orthogonal rotation of axes. Psychometrika, 1946, 11, 51-55.

Manuscript received $8 / 8 / 5 \mathrm{~s}$

Revised manuscript received 11/19/58 